\title{
Réflexions Sur Les Variations Pluviométriques De La Région De Tiaret (Algérie Occidentale) Durant La Période 1984 - 2015
}

\author{
Achir Mohammed \\ Hellal Benchaben \\ Laboratoire de Géomatique et Développement Durable \\ Université Ibn Khaldoun de Tiaret -Algérie-
}

doi: 10.19044/esj.2016.v12n11p498 URL:http://dx.doi.org/10.19044/esj.2016.v12n11p498

\begin{abstract}
The analysis of rainfall is offered over a period of thirty-two years of measurement. This study based on the calculation of a number of indices has to account for the extreme variability of rainfall in the region of Tiaret (western Algeria). It also shows the severe and lasting climate of drought observed in this region from 1984 to 2002. It also highlights the return to a wet phase recorded from 2003 to 2014. The year 2015 has been quite deficient in rainfall. Is it the beginning of a new period of drought?
\end{abstract}

Keywords : Water deficit, rainfall indices, rainfall, climate drought, tiaret

\section{Résumé}

L'analyse de la pluviométrie est proposée sur une période de trentedeux ans de mesure. Cette étude qui s'appuie sur le calcul d'un certain nombre d'indices a permis de rendre compte de l'extrême variabilité des pluies dans la région de Tiaret (Algérie occidentale). Elle montre aussi le caractère sévère et durable de la sécheresse climatique observée dans cette région à partir de 1984 jusqu'à 2002. Elle met aussi en évidence, le retour vers une phase humide notée à partir de 2003 jusqu'à 2014. L'année 2015 s'est montrée plutôt déficitaire en pluviométrie. Serait-elle le début d'une nouvelle période de sécheresse ?

Mots clés : Déficit hydrique, indices pluviométriques, pluviométrie, sécheresse climatique, tiaret

\section{Introduction}

Le changement climatique est maintenant largement reconnu par la communauté scientifique (IPCC, 2013). Certaines études ont montré que ce 
changement se traduit par une intensification des pluies et une succession des évènements extrêmes (New et al., 2001 ; Christensen et al., 2007).

La Rive Sud du bassin méditerranéen s’est caractérisée par la hausse des températures (Nouaceur et al., 2013 ; Donat et al., 2013). Des études menées ont suffisamment montré que la sécheresse a été toujours présente dans l'histoire des pays du Maghreb (Agoumi \& al., 1999, Meddi, Meddi, 2009). L'Algérie a été touchée dans le passé par des périodes d'intenses sécheresses. L’Algérie, dont une bonne partie du territoire est dominée par un climat aride et semi-aride, a plus souffert de cette péjoration pluviométrique récurrente et persistante, aux conséquences souvent dramatiques sur les conditions socio-économiques, notamment rurale. Son impact négatif sur l'agriculture, sur l'alimentation de la nappe phréatique et sur le couvert végétal en constitue un des principaux aspects.

La région d'étude, rattachée administrativement à la wilaya de Tiaret, est enclavée entre l'atlas tellien et l'atlas saharien de l'Algérie occidentale. Les caractéristiques climatiques semblent à première vue disposer cette partie du territoire algérien à des épisodes de sécheresse. Le présent travail s'intéresse ainsi à l'analyse de la série pluviométrique 19842015 par la comparaison des calculs d'un certain nombre d'indices pouvant bien caractériser ses épisodes de sécheresse.

\section{Présentation de la wilaya de Tiaret}

La wilaya de Tiaret, située à l'ouest de pays, couvre une superficie de 20399,10 $\mathrm{km}^{2}$ et s'étend sur une partie de l'Atlas tellien au Nord et sur les hauts plateaux au centre et au Sud. Elle est limitée par plusieurs wilayas à savoir (figure 1) :

les Wilayas de Tissemsilet et Relizane au Nord ;

Laghouat et El-Bayadh au Sud ;

les Wilayas de Mascara et Saida à l'Ouest ;

la Wilaya de Djelfa à l'Est. 


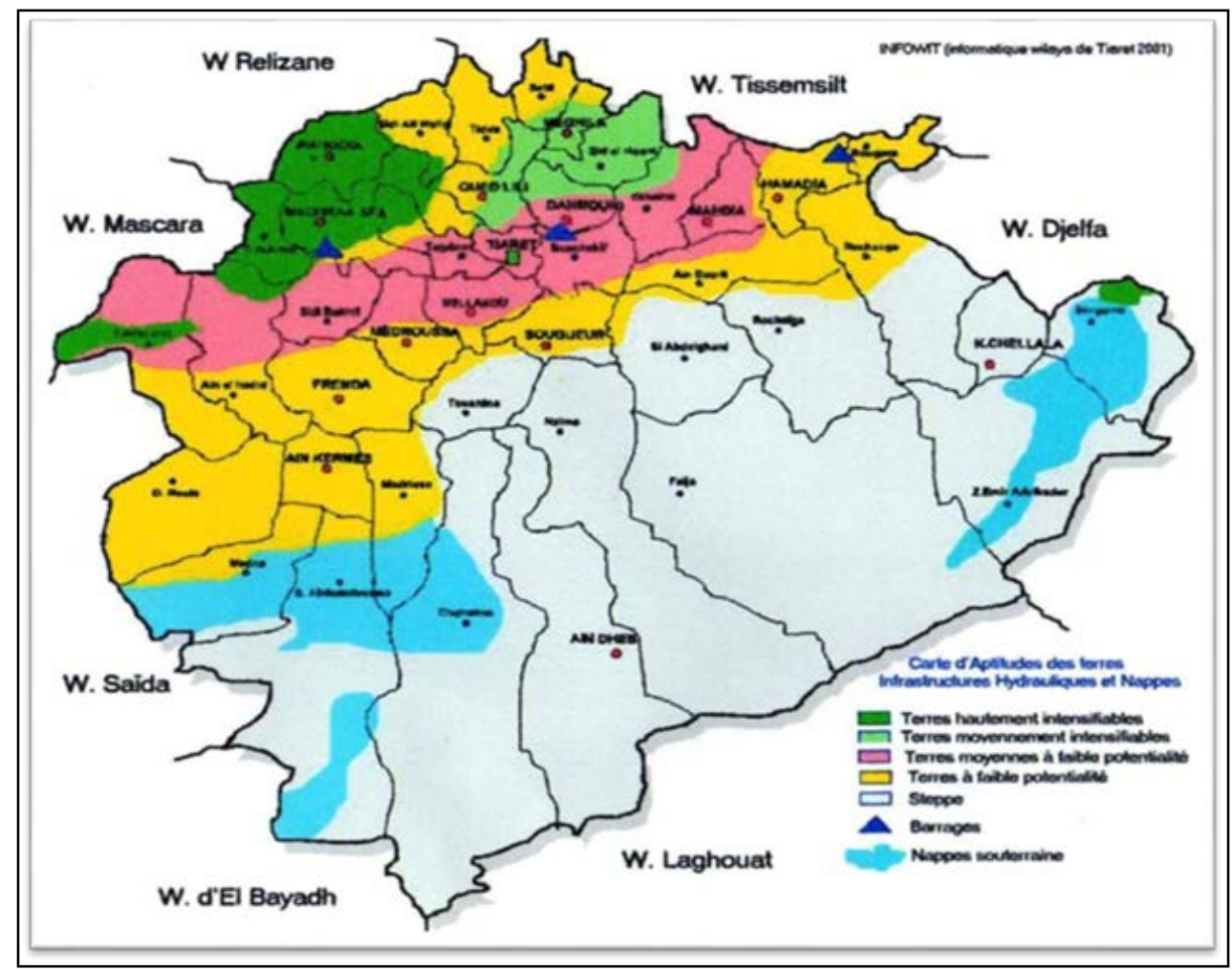

Figure 1 : carte géographique de la wilaya de Tiaret (Algérie occidentale)

Elle se trouve à $1150 \mathrm{~m}$ d'altitude, son climat se caractérise par 02 périodes à savoir : un hiver rigoureux et un été chaud et sec avec une température moyenne de $37,2^{\circ} \mathrm{C}$. Un été chaud et sec avec une température moyenne de $24^{\circ} \mathrm{C}$. En période normale la wilaya de Tiaret reçoit 300 à 400 $\mathrm{mm}$ de pluies par an, avec une fluctuation saisonnière de la pluviométrie allant de $157 \mathrm{~mm}$ en hiver à $31 \mathrm{~mm}$ en été. Elle appartient à l'étage bioclimatique semi-aride inferieur à hiver frais où le climat est du type méditerranéen.

Le relief qui est hétérogène, est matérialisé par : une zone de montage au Nord ;

des hautes plaines au Centre ; des espaces semi-arides au Sud (68,44\%).

La wilaya recèle d'importantes potentialités naturelles et notamment 1.609.900 Ha de terres agricoles, 142.966 Ha de zones steppiques et d'une zone forestière de $142.422 \mathrm{Ha}$. La superficie agricole totale est réparties à raison de 704.596 Ha agricoles utiles dont 14.561 Ha en irrigué et un million d'hectares en steppe, parcours, alfa et forêts. Elle est dominée par le système «céréales- élevage » dont l'intégration constitue l'essentiel de la production agricole et de la croissance économique. 
Le milieu steppique est caractérisé par des altitudes élevées (1100 m en moyenne), les plus hautes steppes atteignent $1300 \mathrm{~m}$ et les plus basses oscillent entre 1000 et $1100 \mathrm{~m}$, ce qui indique que les dénivellations sont peu importantes, soit moins de $200 \mathrm{~m}$. Le substrat est à dominance calcaire. L'espace steppique est caractérisé par l'aridité du climat et la faiblesse des précipitations. Il est très sensible à la désertification où la couverture végétale est fortement dégradée.

\section{Méthodologie}

Pour analyser les valeurs extrêmes, nous utilisons les méthodes issues du calcul d'un certain nombre d'indices.

L’indice SPI "Standardized Precipitation Index » Il a été mis au point par l'université du Colorado en 1990. C'est un indice simple, puissant et souple à la fois, basé sur des données pluviométriques (McKee et al. 1993, 1995).

Il est exprimé mathématiquement (OMM, 2012) comme suit :

\section{SPI $=(\mathbf{P i}-\mathbf{P m}) /$ sigma}

où :

SPI = Indice standardisé de précipitation ;

$\mathrm{pi}=$ Moyenne inter-annuelle (mm) ;

$\mathrm{pm}=$ Moyenne de la série $(\mathrm{mm})$;

sigma = Ecart type de la série $(\mathrm{mm})$.

L'intensité des évènements est évaluée selon la valeur de l'indice obtenu. L'indice SPI indique qu'une sécheresse débute quand sa valeur est inférieure ou égale à $-1,0$ et qu'une sécheresse se termine quand sa valeur devient positive.

L'indice de déficit pluviométrique(IDP) permet aussi de montrer et de calculer le nombre des années déficitaires et leur succession. Une année est qualifié d'humide si cet indice est positif, de sèche lorsqu'il est négatif. Il s'exprime par la formule suivante :

IDP $(\%)=((\mathbf{P i}-\mathbf{P m}) / \mathbf{P m}) \times 100$

où :

IDP : Indice de déficit pluviométrique (en pourcentage) ;

$\mathrm{Pi}$ : précipitation annuelle (en $\mathrm{mm}$ ) ;

$\mathrm{Pm}$ : précipitation moyenne (en $\mathrm{mm}$ ).

L’Indice de pluviosité (Ip) est le rapport de la hauteur de précipitation d'une année à la hauteur moyenne annuelle de précipitation de la série. Il est exprimé par la formule suivante :

\section{$\mathbf{I p}=\mathbf{P i} / \mathbf{P m}$}

Une année est dite humide si ce rapport est supérieur à 1 et sèche s'il est inférieur à 1 . 
L'indice de sécheresse (IS) permet d'estimer le déficit pluviométrique annuel. Cet écart à la moyenne est la différence entre la hauteur des précipitations d'une année Pi et la hauteur moyenne annuelle de précipitations $\mathrm{P}$ de la série. La formule est :

IS $=\mathbf{P i}-\mathbf{P}$

L'indice est positif pour les années humides et négatives pour les années sèches.

\section{Résultats}

\section{Précipitations moyennes annuelles}

L'analyse de la série pluviométrique montre nettement la variabilité interannuelle de la pluviométrie et sa tendance à la baisse, en plus d'une dispersion croissante dans le temps qui a pour origine la grande irrégularité des épisodes excédentaires et déficitaires (tableau 1).

Tableau 1 : Données pluviothermique de Tiaret (période 1984-2015)

\begin{tabular}{|c|c|c|c|c|c|c|c|c|c|c|c|c|}
\hline Mois & Janv & Févr & Mars & Avril & Mai & Juin & Juillet & Aout & Sept & Oct & Nov & Déc \\
\hline Pmm & 39,9 & 39,9 & 35,1 & 35,9 & 28,2 & 11,8 & 3,4 & 9,4 & 33,0 & 31,3 & 42,8 & 35,5 \\
\hline $\mathrm{T}^{\circ} \mathrm{C}$ & 6,1 & 7,0 & 9,5 & 11,9 & 16,3 & 21,9 & 26,3 & 26,1 & 21,3 & 16,6 & 10,6 & 7,3 \\
\hline
\end{tabular}

La période 1984-2015 a enregistré des tranches pluviométriques en dessous de la moyenne $(\operatorname{Pr}$ moy $=342,6 \mathrm{~mm})$ durant une quinzaine d'années; les dix-sept autre étaient plus ou moins humides. Elle s'est caractérisée respectivement par une sécheresse légère à modérée et une humidité légère à modérée. Elle est marquée par une période sèche de sept mois, une température minimale de $-3,2^{\circ} \mathrm{C}$ et une température maximale de $37,2{ }^{\circ} \mathrm{C}$.

\section{Indices climatiques}

\section{Indice de précipitations standardisé}

L'illustration graphique de l'indice de précipitations standardisé, à partir de la série 1984-2015, a permis de mettre en évidence deux périodes bien distinctes; la première étant sèche tandis que la seconde est plutôt humide (figure 2). 


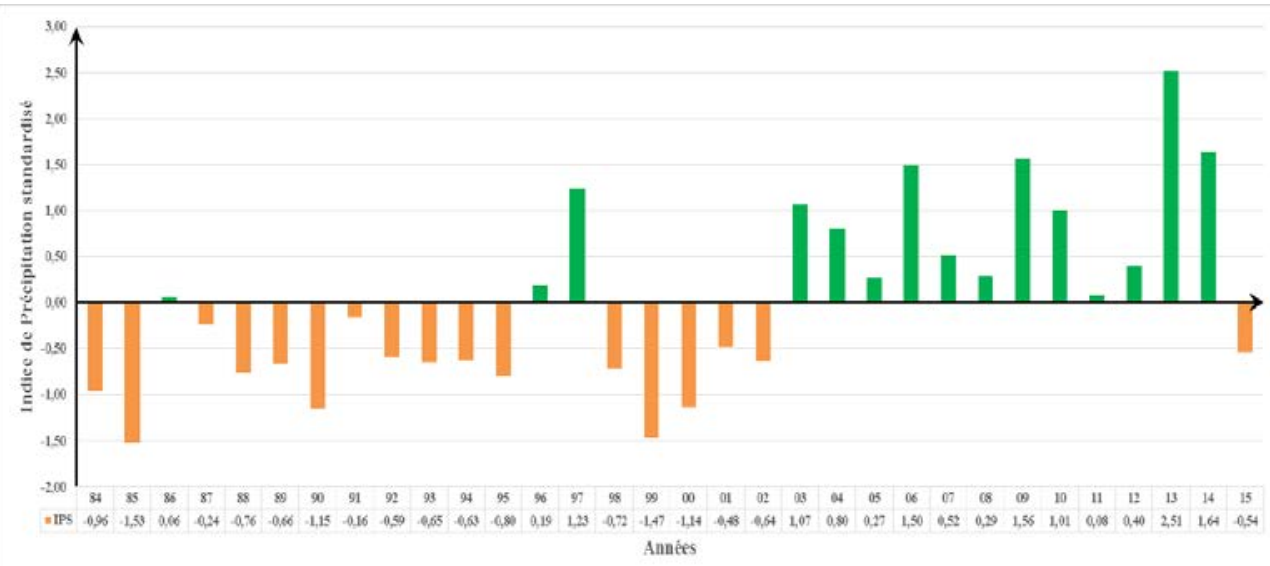

Figure 2 : Indice de Précipitations Standardisé (SPI) durant la période 1984-2015.

Les trente-deux années d'observation, de la série pluviométrique 1984-2015, sont réparties en années sèches et en années humides en tenant compte des valeurs de l'indice de précipitations standardisé (tableau 2).

Tableau 2 : Fréquences des années pluviométriques en fonction de l’Indice de Précipitations Standardisé (SPI)

\begin{tabular}{|c|c|c|c|c|c|}
\hline Echelle SPI & Observation & $\begin{array}{c}\text { Nombre } \\
\text { d'années }\end{array}$ & Echelle SPI & Observation & $\begin{array}{c}\text { Nombre } \\
\text { d'années }\end{array}$ \\
\hline $2<$ SPI & $\begin{array}{c}\text { Humidité } \\
\text { extrême }\end{array}$ & 01 & $0<\mathrm{SPI}<-0.99$ & $\begin{array}{c}\text { Sécheresse } \\
\text { légère }\end{array}$ & 13 \\
\hline $1.5<\mathrm{SPI}<1.99$ & Humidité sévère & 03 & $-1<\mathrm{SPI}<-1.49$ & $\begin{array}{c}\text { Sécheresse } \\
\text { modérée }\end{array}$ & 03 \\
\hline $1<\mathrm{SPI}<1.49$ & $\begin{array}{c}\text { Humidité } \\
\text { modérée }\end{array}$ & 03 & $\begin{array}{c}-1.5<\mathrm{SPI}<- \\
1.99\end{array}$ & $\begin{array}{c}\text { Sécheresse } \\
\text { sévère }\end{array}$ & 01 \\
\hline $0<\mathrm{SPI}<0.99$ & Humidité légère & 08 & $\mathrm{SPI}<-2$ & $\begin{array}{c}\text { Sécheresse } \\
\text { extrême }\end{array}$ & 00 \\
\hline
\end{tabular}

La série pluviométrique 1984-2015 est caractérisée par treize années de sécheresse légère et huit ans d'humidité légère. L'année 1985 a connu une sécheresse sévère $(P m m=153,4 \mathrm{~mm})$ et l'année 2013 s'est manifestée par une humidité extrême avec une pluviométrie de 662,2 mm. La série a aussi connu trois années chacune de sécheresse et d'humidité sévères. Il est à noter que la période 1984-2015 n’a pas connue de sécheresse extrême.

\section{L’indice de déficit pluviométrique}

L'indice de déficit pluviométrique a permis d'estimer la variation ponctuelle des précipitations par rapport à la normale. Le déficit le plus important enregistré durant toute la période d'observation est de - 55,7\% (figure 3 a). Le déficit pluviométrique est plus important, en nombre d'années successives, de 1984 à 1985, de1987 à 1995 et de 1998 à 2002. La première phase de déficit pluviométrique s'est étalée sur neuf ans avec un 
taux maximal de - 41,8\%. La seconde phase est marquée par cinq années de déficit pluviométrique et un taux maximal de - 53,5\%. L'excès pluviométrique, pour cette série 1984-2002 est observé en 1986, 1996 et 1997 avec respectivement $02 \%, 07 \%$ et $44,8 \%$ (figure3 b).

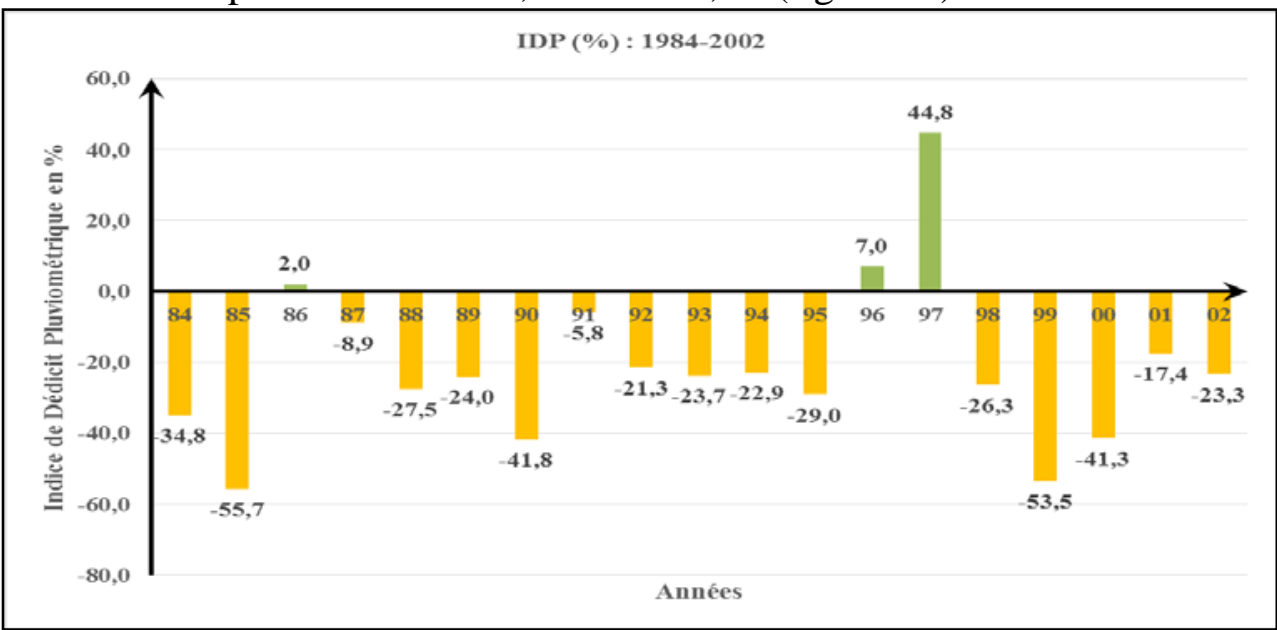

Figure 3 a : Indice de Déficit Pluviométrique (IDP \%) durant la période 1984-2002.

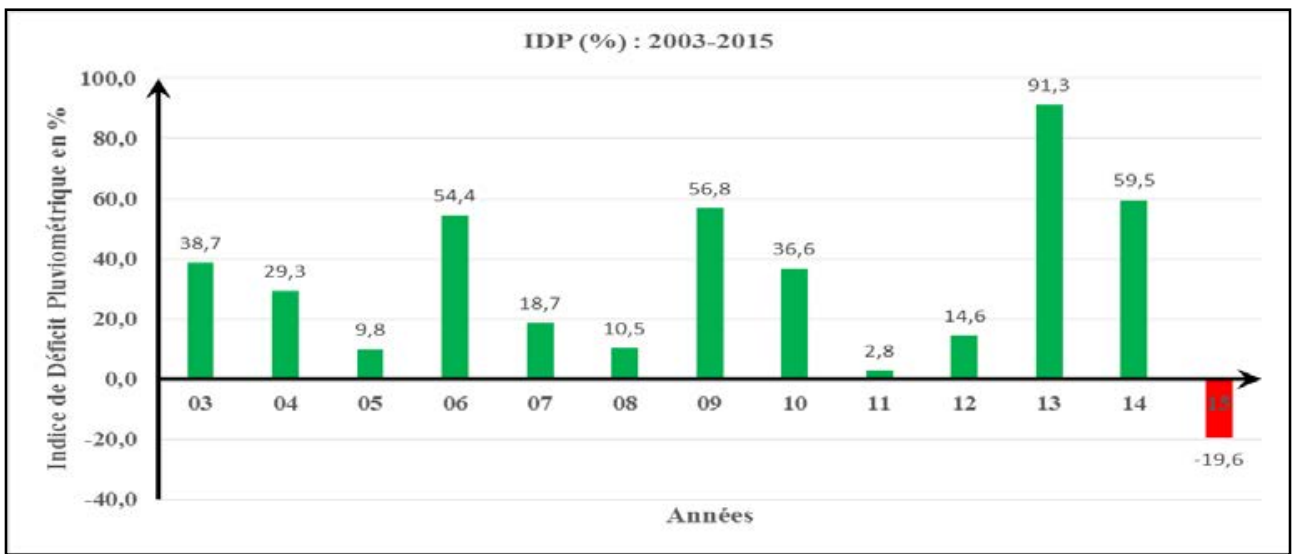

Figure 3 b : Indice de Déficit Pluviométrique (IDP \%) durant la période 2003-2015.

Les treize dernières années de la série 1984-2015 se sont caractérisées par un excès pluviométrique maximal de 91,3\% en 2013 (figure3 b); l'année 2014 a totalisé un taux de 59,5\%. L'excès pluviométrique s'est répandu sur toute la période d'observation mis à part l'année 2015 qui a enregistré un déficit pluviométrique de - 19,6\%.

Les années humides, au nombre de douze, se sont réparties en deux groupes. Le premier groupe est formé de quatre années avec un excès pluviométrique de plus de $50 \%$; le deuxième s'est étalé sur huit ans avec des taux allant de 2,8\% à 38,7\%(figure3 b). 


\section{Indice de pluviosité}

Le graphique de l'évolution de l'indice de pluviosité a permis de constater que les épisodes de sécheresse de 1984 à 1995 et de 1998 à 2002 sont d'une intensité allant de légère à modérée (figure 4). La succession des années humides ont fait suite à celle des années sèches depuis 2003 jusqu’à 2014.

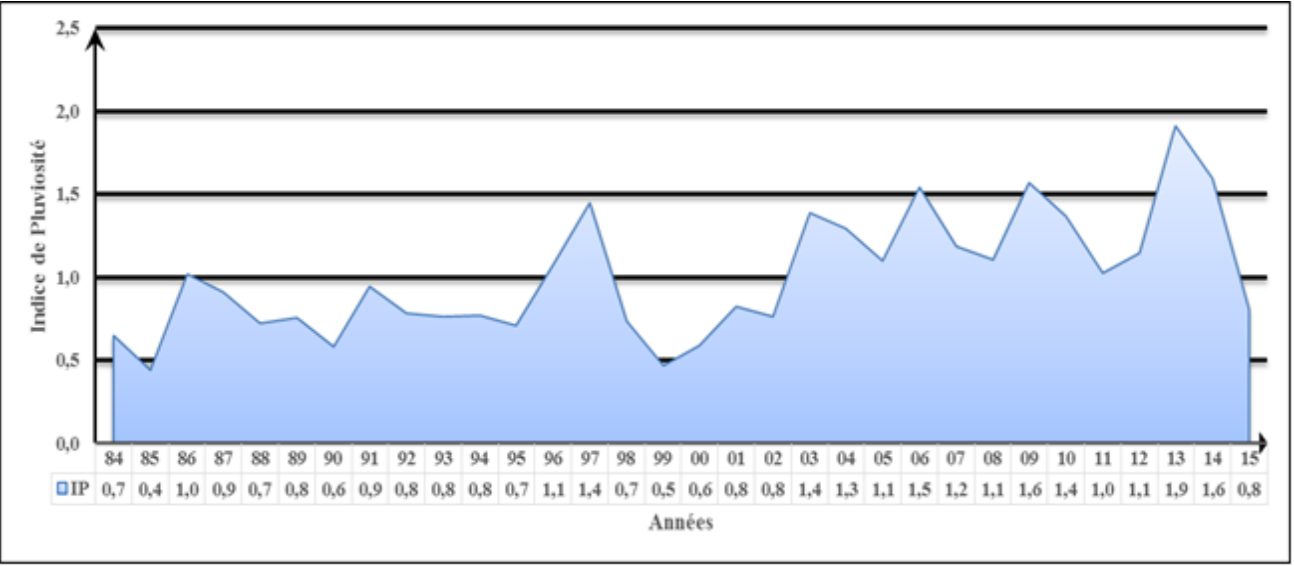

Figure 4 : Indice de Pluviosité (IP) durant la période 1984-2015.

\section{Indice de sécheresse}

La détermination du niveau de sévérité des sécheresses vécues est effectuée par le calcul de l'indice de l'écart à la moyenne. L'analyse de la figure 5 a rendu encore possible la confirmation d'une sécheresse légère à modérée observée dans la série pluviométrique 1984-2015.

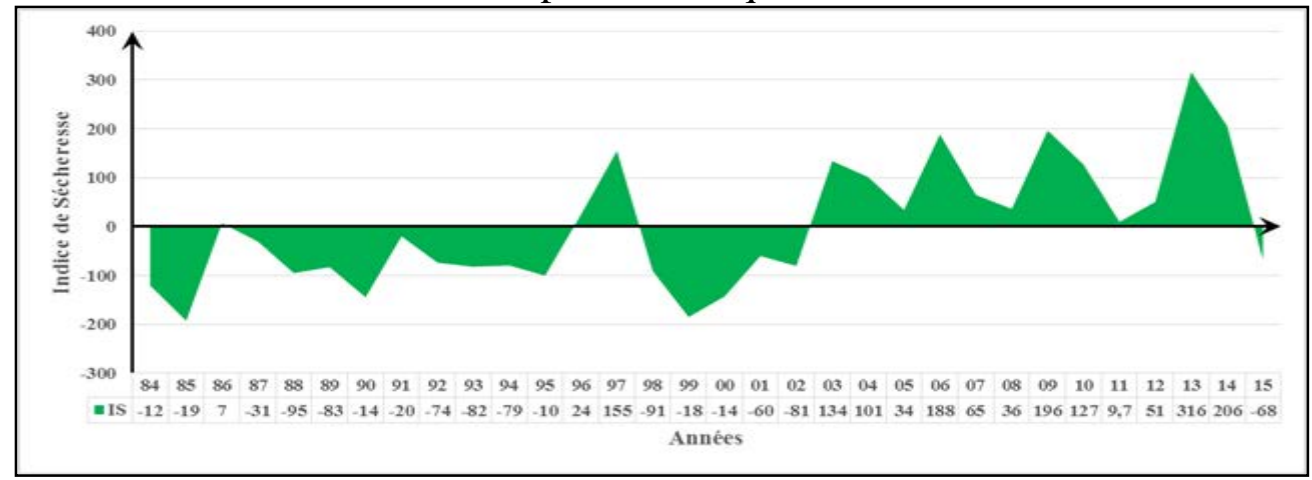

Figure 5 : Indice de Sécheresse durant la période 1984-2015.

Les épisodes de sécheresse sont de plus en plus longues où elles s'étalent sur neuf à dix années consécutives. La répétition des années sèches s’est accélérée et la longévité de la période sèche également. 


\section{Discussion}

Les conditions climatiques de l'Algérie du Nord se caractérisent par des pluies concentrées sur la saison fraîche à jours courts avec de longues sècheresses estivales (Seltzer, 1946 ; Emberger, 1955). Les précipitations exercent une action prépondérante pour la définition de la sècheresse globale du climat (Le Houerou et al., 1977).

En écosystème steppique, défini comme la portion du territoire national délimité au Nord par l'isohyète 400 mm et au Sud par l'isohyète 100 $\mathrm{mm}$, les fluctuations des précipitations sont sévères. Certaines années sont très déficitaires en pluviométries tandis que d'autres sont humides.

L'analyse des précipitations, par des indices, a montré, qu'après les déficits pluviométriques de la période 1984-1995, une phase beaucoup plus humide est en train de se manifester. Les signes de changements se sont apparues dans la production céréalière enregistrée entre 2003 et 2014. L'alternance brutale des années sèches et humides, observée au sahel, a tendance à s'installer dans les pays du Maghreb dont l'Algérie (Abdou, 2010 ; Dieppois et al., 2010 ; Nouaceur et al, 2013).

Les résultats obtenus pour la région de Tiaret de l'Algérie occidentale ont mis en évidence en plus des deux périodes caractéristiques sèches (19842002) et humides (2003-2014), des courtes périodes pluviométriques excédentaires ou déficitaires sur la série pluviométrique 1984-2015. Les épisodes de sécheresse de 1984 et 1995 sont aussi observées dans la steppe du Sud de Tlemcen (Benabadji et Bouazza, 2000).

L'évolution des pluies est marquée de particularités bien distinctes. L'analyse des données climatiques enregistrées sur trente-deux années montre l'amorce de nouvelles conditions pluviométriques en opposition avec les périodes de sécheresse passées (Nouaceur, 2011). Cette constatation va dans le sens d'une augmentation de la fréquence des pluies intenses qui ont vraisemblablement un effet moindre sur la recharge des nappes phréatiques que sur l'amplification du ruissellement sur les versants, des crues et des inondations (Medjerab, 2007).

\section{Conclusion}

Les résultats des analyses pluviométriques effectuées montrent que la région Tiaret (Algérie occidentale) est passée par des épisodes de sécheresse et d'humidité. Cette étude qui s'appuie sur le calcul d'un certain nombre d'indices a permis de se rendre compte de l'extrême variabilité des pluies. Elle a montré aussi le caractère sévère et durable de la sécheresse climatique observée dans cette région de 1984 à 2002. Elle a mis aussi en évidence, le retour vers un épisode humide de 2003 à 2014. 


\section{References:}

Abdou A. 2010. Variabilité et changements du climat au Sahel : ce que l'observation nous apprend sur la situation actuelle. Grain de Sel 2010 ; 49 : 13-14.

Agoumi, A, Senoussi S, Yacoubi M, Fakhredine A, Sayouti E.H, Mokssit A et Chikri N. 1999. Changements climatiques et ressources en eau. Hydrogéologie Appliquée, vol. 12, n 11, 163-182.

Ayache A, Ayad N, Hellal B, Benhanifia K et Gacemi M.A. 2015. Study of the temporal evolution of land use in the steppe south of Tlemcen province for the period 1987 and 2010 using remote sensing and GIS thechnique (Western Algeria). International Journal of Remote Sensing \& Geoscience (IJRSG), Volume 4, Issue 4: 28-34.

Ayad N, Hellal B et Maatoug M'H. 2007. Dynamique des peuplements d'Artemisia herba-alba Asso dans la steppe du Sud oranais (Algérie occidentale). Revue Sécheresse, 18 (3) : 193 - 8.

Benabadji N. et Bouazza M. 2000. Contribution à une étude bioclimatique de la steppe à Artemisia herba-alba Asso dans l'Oranie (Algérie occidentale). Sécheresse. Vol 11, $\mathrm{N}^{\circ}$ 2, 117-23.

CFT., 2014 -Conservation des forêts de la Wilaya de TIARET- Service de cartographie et Service des statistiques.

Christensen JH, Hewitson B, Busuioc A, Chen A, Gao X, Held I et al., 2007. Regional Climate Projections. In : Climate Change 2007: The physical Sciences Basis. Contribution of Working Group I to the Fourth Assessment Report of the Intergovernmental Panel on Climate Change, Solomon S, Qin D, Manning M, Chen Z, Marquis M, Averyt KB, Tignor M and HL Miller (eds.) Cambridge University Press : Cambridge, New York, 847-940.

Dieppois B, Durand A, Fournier M, Massei N et Hassane B. 2010. Relations entre la pluviométrie au Sahel et divers indices climatiques sur l'Atlantique : exemple de la station de Maïnesoroa (Niger) entre 1950 et 2005. Actes du XXIII ème colloque international de climatologie, Rennes.

Donat M.G, Peterson T.C, Brunet M, King A.D, Almazroui M et Kolli R.K. 2013. Changes in extreme temperature and precipitation in the arab region: long-term trends and variability related to ENSO and NAO. International Journal of Climatology, DOI: 10.1002/joc.3707.

DSA., 2014 -Direction des Services Agricoles, Wilaya de TIARET- Service des statistiques.

Emberger L. 1955. Une classification biogéographique des climats. Trav. Lab. Bot. Zool. Fac. Sci.Serv. Montpellier 7, p 3-43.

Hellal B, Ayad N, Maatoug M'H et Boularas M. 2007. Influence du fatras sur la biomasse foliaire de l'alfa (Stipa tenacissima L) des hautes plaines steppiques du sud Oranais (Algérie occidentale). Revue Sécheresse ; 18 (1) : $65-71$. 
IPCC (Intergovernmental Panel on Climate Change) GIEC. 2013. Changements climatiques en 2013, Les éléments scientifiques, résumé à l'intention des décideurs, service d'appui technique du groupe de travail I GTI,

Le-Houerou H.N, Claudin J et Pouget M. 1977. Etude bioclimatique des steppes algériennes avec une carte bioclimatique au 1/1000.000. Bull. Soc. Hist. Nat. Afri. Nord. 36-40.

Mckee T.B, Doesken N.J and Kleist J. 1995. Drought Monitoring with Multiple Times Scales. American Meteorological Society, $9^{\text {th }}$ Conference on Applied Climatology, 15-22 Janvier, Dallas, TX, pp. 233-236.

MCKee T.B, Doesken N.J and Kleist J. 1993. The relationship of drought frequency and duration to time scales, Proceedings of the 8th Conference on Applied Climatology, 17-22 January 1993, Anaheim, California, 179-184.

Meddi $\mathrm{H}$ et Meddi M. 2009. Variabilité des précipitations annuelles du Nord-Ouest de l'Algérie. Sécheresse, vol. 20, n 1, 57-65.

MEDJERAB A. 2007. Les situations pluviométriques extrêmes dans le nordouest de l'Algérie. In : Climat, tourisme, environnement, Actes du XXème colloque de l'Association Internationale de Climatologie (Carthage, Tunisie), H. BEN BOUBAKER édit., 381-386.

Nouaceur Z, Laignel B, Turki I., 2013 : Changements climatiques au Maghreb : vers des conditions plus humides et plus chaudes sur le littoral algérien ? Physio-Géo, Volume 7,

Nouaceur Z. 2011. Vers un retour des pluies sur la rive-Sud du Bassin méditerranéen occidental : analyse et évaluation de la tendance pluviométrique sur plus d'un demi-siècle. The Annals of Valahia University of Targoviste, Romania, Géographical Series 11: 32-6.

New M, Todd M, Hulme M and Jones P. 2001. Precipitation measurements and trends in the twentieth century. International Journal of Climatology, 21(15), 1889-1922.

O.M.M. 2012, Guide d'utilisation de l'indice de précipitations normalisé, $\mathrm{OMM} \mathrm{n}^{\circ} 1090$. 